\title{
Adrenalectomía laparoscópica. Consideraciones a propósito de 24 procedimientos
}

\author{
Pascual Piédrola JI, Cuesta Alcalá JA, Grasa Lanau V, Labairu Huerta L, Napal Lecumberri S, \\ Ipiens Aznar AP.
}

Servicio de Urología. Hospital de Navarra. Pamplona.

Actas Urol Esp. 2007;31(2):98-105

\section{RESUMEN}

ADRENALECTOMÍA LAPAROSCÓPICA. CONSIDERACIONES A PROPÓSITO DE 24 PROCEDIMIENTOS

Introducción y objetivos: La adrenalectomía laparoscópica ha ido ganando adeptos desde la publicación del primer caso en 1992. Actualmente se ha convertido en la técnica de elección en el tratamiento quirúrgico de la suprarrenal. Nuestro objetivo en el presente trabajo es mostrar la experiencia adquirida en el manejo de esta técnica laparoscópica y aportar algunos consejos prácticos para su correcto abordaje.

Material y Método: Entre mayo de 1998 y agosto de 2006 realizamos un total de 24 adrenalectomías laparoscópicas en 22 pacientes (15 mujeres, 7 varones). 13 fueron derechas y 11 izquierdas ( 2 pacientes de forma bilateral: 1 MEN II, 1 hiperplasia cortical bilateral), con edades comprendidas entre 24-78 años (media 49,5 $\pm 14,3$ ). El diagnóstico clínico fue: feocromocitoma ( $\mathrm{n}=10)$, Cushing (n=6), Conn (n=4), metástasis pulmonar $(\mathrm{n}=2)$, incidental $(\mathrm{n}=2)$. La posición en todos los casos fue de decúbito lateral completo con Pillet para abrir el espacio costo-ilíaco. En el lado derecho se utilizaron 4 trocares y tres en el izquierdo. El acceso abdominal lo realizamos con trocar de Hasson por medio de minilaparotomía, no empleando la aguja de Veress para el neumoperitoneo, que manteníamos con una presión inferior a 12 $\mathrm{mmHg}$.

Resultados: Hubo 1 reconversión a cirugía abierta. El tiempo operatorio para toda la serie osciló entre 59 y 400 min. (media $182,5 \pm 97,75$ ). En los primeros 12 casos el tiempo quirúrgico medio fue de $261,6 \pm 76,61$ minutos y en los últimos 12 de $103,3 \pm 20,92$ minutos $(\mathrm{p}<0,001)$. El diámetro tumoral osciló entre 1,3 y $6 \mathrm{~cm}$ (media 3,08 $\pm 1,25)$ con un peso entre 8 y $92 \mathrm{~g}$ (media 30,13 \pm 21 ). El sangrado fue inferior a $100 \mathrm{cc}$ en todos los casos (10-100 cc, media 43,26 \pm 25$)$ menos en uno que fue de $600 \mathrm{cc}$. Complicaciones intraoperatorias hubo en dos casos, una perforación hepática por separador laparoscópico al principio de la serie y una laceración capsular del bazo, ambas resueltas intracorpóreamente. Los tipos histológicos fueron: 9 adenomas corticales, 9 feocromocitomas, 3 hiperplasias nodulares, 2 metástasis de carcinoma de pulmón y 1 pseudoquiste suprarrenal. El alta hospitalaria se produjo entre 3 y 5 días (media 3,62 $\pm 0,82$ ), con una diferencia significativa $(\mathrm{p}<0,001)$ entre los 12 primeros y los 12 últimos.

Conclusiones: El abordaje laparoscópico de la adrenal es actualmente el tratamiento de elección en los tumores suprarrenales, con unas contraindicaciones relativas en tumores malignos o mayores de $7 \mathrm{~cm}$ de diámetro. La curva de aprendizaje es obligatoria, siendo algo menor en el caso de la localización derecha que en la izquierda.

Palabras clave: Adrenal. Laparoscopia. Técnica quirúrgica.

\section{ABSTRACT}

LAPAROSCOPIC ADRENALECTOMY. REFLECTIONS AFTER 24 PROCEDURES

Introduction and objectives: Laparoscopic adrenalectomy has gained rapid recognition since publication of the first case in 1992. Currently it is the technique of choice for the surgical treatment of the adrenal gland. Our objective in this paper is to share our experience with this technique and offer some practical advice on how to approach it.

Materials and Methods: Between May 1998 and August 2006 we did a total of 24 laparoscopic adrenalectomies in 22 patients (15 men, 7 women). The right gland was removed in 13 cases and the left in 11. Surgery was bilateral in two cases (one was MEN II, the other bilateral cortical hyperplasia). Average age was $49.5 \pm 14.3$ years (range 24 to 78 ). Clinical diagnosis was: Pheochromocytoma $(n=10)$, Cushing $(n=6)$, Conn $(n=4)$, metastases from lung carcinoma $(n=2)$ and non-functioning tumor $(n=2)$. For surgery, all patients were in total lateral decubitus with a pillow to increase the costal-iliac space. We used four trocars on the right side and three on the left. Abdominal access was by Hasson trocar after minilaparotomy. We kept pneumoperitoneal pressure below $12 \mathrm{mmHg}$; a Veress needle was not used for this procedure.

Results: Open surgery was required in one case. Time operation was between 59 and 400 minutes (mean $182 \pm 98$ min.). In the first 12 cases average time was $261 \pm 77$ minutes and in the final 12 cases was $103 \pm 21$ minutes $(\mathrm{p}<0.001)$. Tumour diameter was between 1.3 and $6 \mathrm{~cm}$ (mean $3.08 \pm 1.25 \mathrm{~cm}$ ) and tumour weight was between 8 and $92 \mathrm{~g}$ (mean $30.13 \pm 21 \mathrm{~g}$ ). Except in one case with $600 \mathrm{ml}$ blood loss, bleeding was less than $100 \mathrm{ml}(\mathrm{n}=23$, range: $10-100$, mean $43.26 \pm 25 \mathrm{ml})$. We only had intraoperative complications in two cases: perforation of the liver by the laparoscope retractor (at the beginning of the series) and injury to the spleen capsule. Both complications were resolved laparoscopically. Cases by histologic type were: nine cortical adenomas, nine pheochromocytomas, three nodular hyperplasias, two metastases from lung carcinoma, and one adrenal pseudocyst. Discharge from hospital was between three and five days (mean $3.62 \pm 0.82)$ with a statistical difference $(\mathrm{p}<0.001)$ between twelve first cases and the last ones.

Conclusions: The adrenal laparoscopic approach is currently the technique of choice for removing adrenal tumours although with malign tumours or over $7 \mathrm{~cm}$ in diameter there are some contraindications and disadvantages relative to open surgery. There is inevitably a learning curve but satisfactory results are quickly attainable.

Keywords: Adrenal. Laparoscopy. Surgical technique. 
$\mathrm{L}^{\mathrm{a}}$ cirugía laparoscópica ha supuesto una uténtica revolución en el tratamiento quirúrgico de la patología tumoral de las glándulas adrenales. Desde la primera descripción en 1992 por Gagner ${ }^{1}$, el abordaje laparoscópico de las adrenales se ha convertido en el patrón de oro en el tratamiento de los tumores suprarrenales. La adrenalectomía laparoscópica estaría indicada en todos aquellos casos con masas suprarrenales funcionantes como los aldosteronomas (enf. de Conn), adenomas (enf. de Cushing), mielolipomas, feocromocitomas y otras patologias benignas. También estaría indicada en aquellos tumores no funcionantes descubiertos de forma incidental. La presencia de metástasis adrenales sería una indicación para la cirugía laparoscópica siempre que se analicen individualmente los casos en función del tipo de tumor primario, tamaño y posibilidad de compromiso de estructuras vecinas en el TAC o RMN.

La localización retroperitoneal de las adrenales hace que puedan ser abordadas por vía transabdominal o retroperitoneal, ambas con sus ventajas e inconvenientes, aunque la decisión final sobre la vía elegida la dará la preferencia y experiencia del cirujano en cada una de éstas.

Al igual que en otras patologías, el abordaje laparoscópico de las adrenales tiene la ventaja contrastada de un menor dolor postoperatorio, herida quirúrgica mínima, con el consiguiente beneficio de una menor cicatriz, estancia más corta que en los procedimientos abiertos y menor tiempo quirúrgico una vez superada la curva de aprendizaje.

Queremos mostrar en este trabajo la experiencia que hemos adquirido en el abordaje laparoscópico transperitoneal de las glándulas adrenales que comenzamos a realizar en el Servicio de Urología del Hospital de Navarra en 1998.

\section{MATERIAL Y MÉTODO}

Entre mayo de 1998 y agosto de 2006 hemos realizado 24 adrenalectomías laparoscópicas transperitoneales en 22 pacientes (15 mujeres y 7 varones), 13 derechas y 11 izquierdas. En dos pacientes se realizó adrenalectomía bilateral en el mismo tiempo quirúrgico (1 MEN II, 1 hiperplasia cortical bilateral). La edad media de los pacientes fue 49,5 (14,3) años (rango: 24-78). Las indica- ciones quirúrgicas por patologías fueron: feocromocitoma $(n=10)$, Cushing $(n=6)$, Conn $(n=4)$, metástasis pulmonar $(n=2)$, tumor no funcionante/incidental $(n=2)$.

En todos los pacientes se obtuvo el preceptivo consentimiento informado, realizando una preparación mecánica del intestino desde 24 horas antes de la cirugía que incluyó dieta líquida y laxantes (solución de Goliteli,4 1). Se administró profilaxis tromboembólica con heparina de bajo peso molecular, cruzando para reserva $2 \mathrm{U}$. de sangre compatible. El mismo día se administró una dosis de antibiótico de amplio espectro (cefalosporina de $3^{\underline{a}}$ generación). Se administraron $\alpha$ bloqueantes y antagonistas del calcio (fenoxibenzamina, propanolol, nifedipino) en el caso de feocromocitomas, ketoconazol en la enfermedad de Cushing y espironolactonas en hiperaldosteronismo primario (enfermedad de Conn).

El material empleado incluyó óptica laparoscópica de $0^{\circ}$ y $30^{\circ}$, piecerío laparoscópico (pinzas, tijera, disector), caja de minilaparotomía, 1 trocar de Hasson , 1 trocar de $10 \mathrm{~mm}$ y 1-2 trocares de $5 \mathrm{~mm}$ (según fuese la adrenal izquierda o derecha), bisturí armónico (Ultracission $\AA$, Ethicon Inc., USA), endoclips vasculares (Hem-o-Lok ${ }^{\circledR}$, Teleflex, NC, USA), pinzas de coagulación bipolares, aspirador y bolsa endoscópica así como caja de cirugía abierta con material dentro del quirófano. La posición quirúrgica era en decúbito lateral con Pillet, colocando antes de la intervención sonda vesical y nasogástrica.

La localización de los puertos de trabajo vienen indicados en la Figura 1. En el caso de adrenalectomía bilateral el trocar de Hasson se colocó dos o tres dedos por encima del ombligo, manteniéndolo para ambos lados y protegiéndolo con adhesivo estéril para el cambio postural, siendo la disposición del resto de los trocares similar a la descrita previamente (Fig. 2). La técnica quirúrgica fue la habitual para estos procedimientos, destacando en el lado derecho la colocación de una pinza de agarre para separar adecuadamente el hígado (Fig. 3) y evitar así la utilización de separadores laparoscópicos se pudiesen producir lesiones en la cápsula del hígado. La vena adrenal era siempre seccionada entre clips (Fig 4). En ocasiones la salida de la vena suprarrenal se localizaba en una posición 


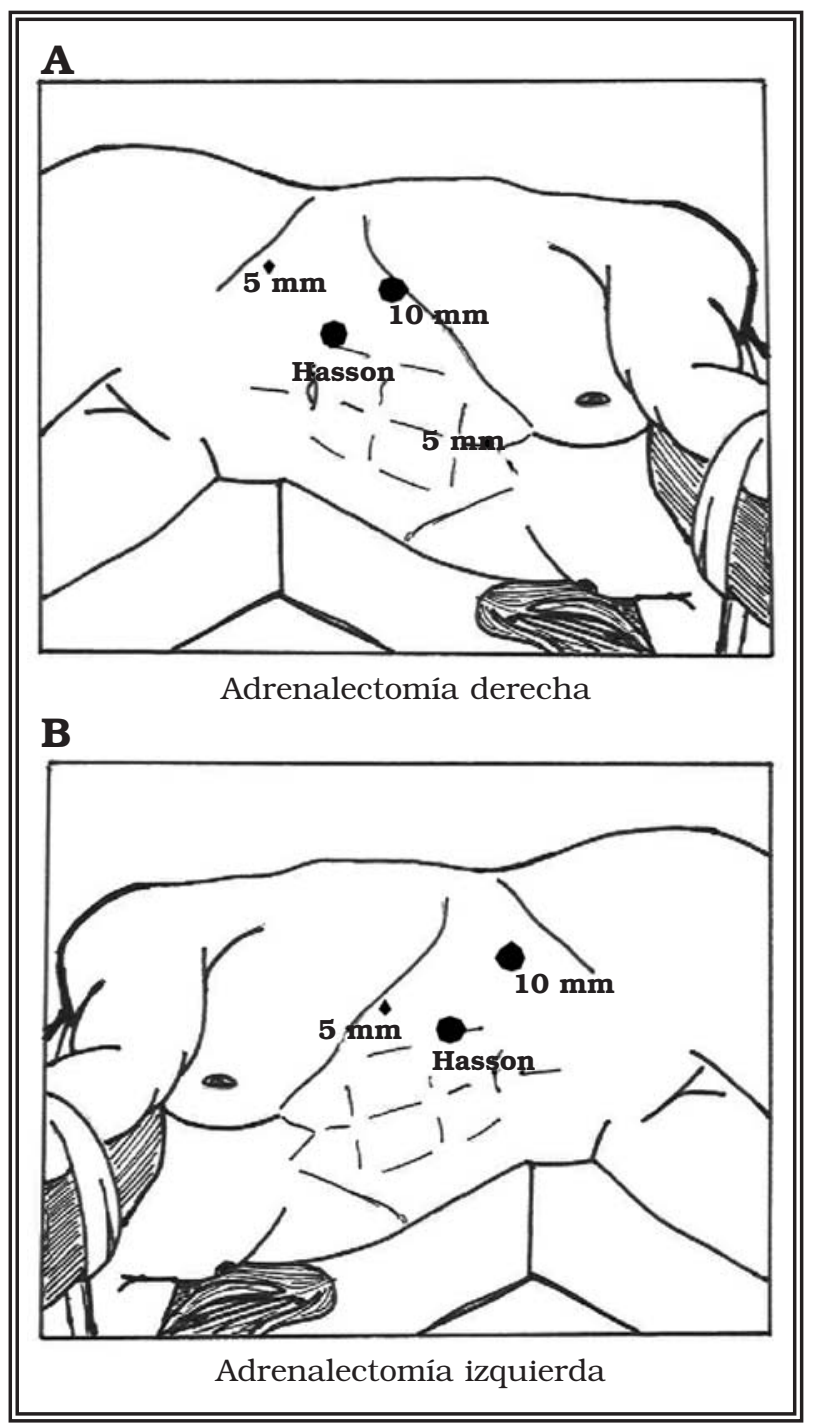

FIGURA 1. En (A) disposición de los puertos de trabajo en la adrenalectomía laparoscópica derecha. En (B) se muestra la posición en el lado izquierdo (modificado de ref. 16).

posterior a la pared lateral de la cava siendo de gran utilidad en estos casos la óptica de $30^{\circ}$ (Fig. 5). En la adrenalectomía izquierda utilizamos de forma sistemática 3 puertos de trabajo, gracias a la reflexión medial del bazo que se produce al seccionar el ligamento freno-esplénico, evitando así la colocación de otro puerto accesorio y la utilización del retractor laparoscópico (Fig. 6). En los casos de metástasis adrenal, donde el tejido graso peri-adrenal suele presentar un aspecto edematoso o pseudo-inflamatorio fue de gran utilidad el empleo del bisturí armónico (Fig. 7).

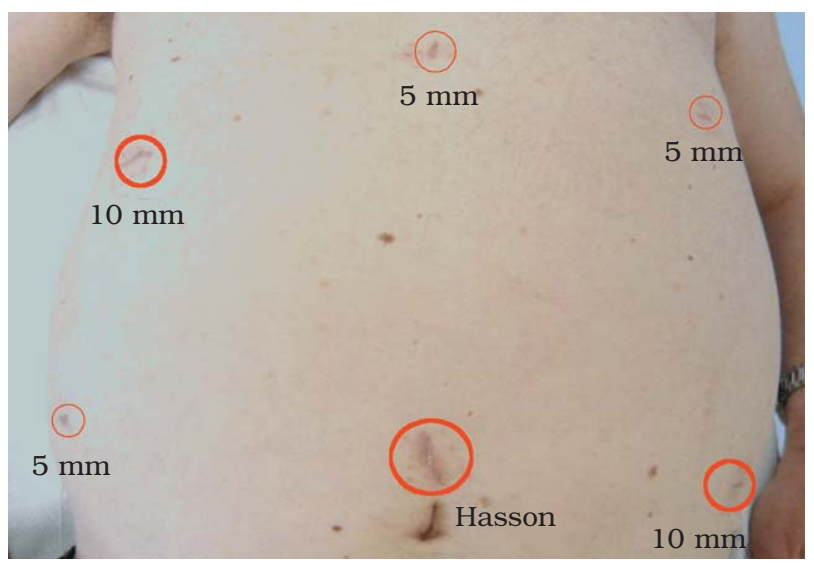

FIGURA 2. Disposición de los puertos de trabajo en un caso de adrenalectomía laparoscópica bilateral.

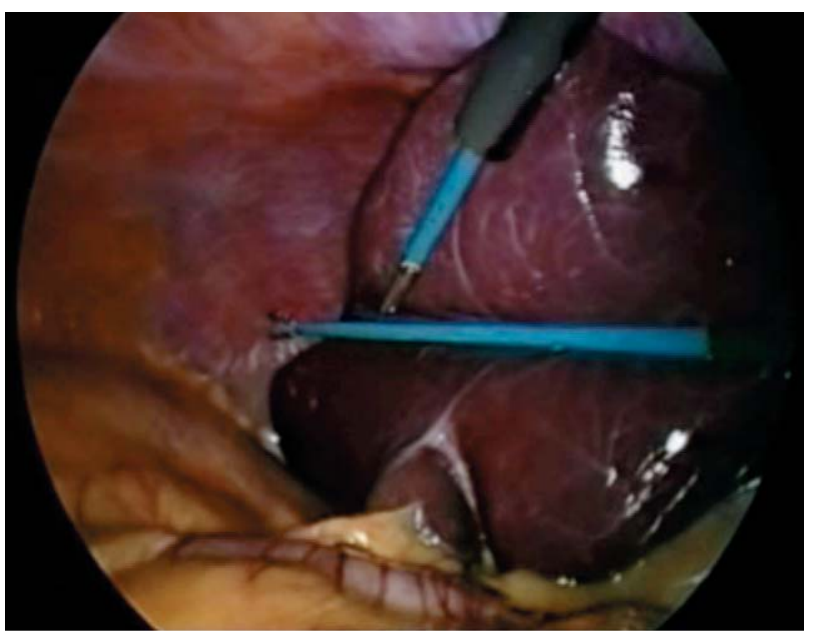

FIGURA 3. Maniobra de separación laparoscópica de una lengüeta hepática prominente con la ayuda de una pinza de agarre colocada a través del puerto de $5 \mathrm{~mm}$ a nivel xifoideo. Obsérvese la excelente exposición que nos permite del campo quirúrgico.

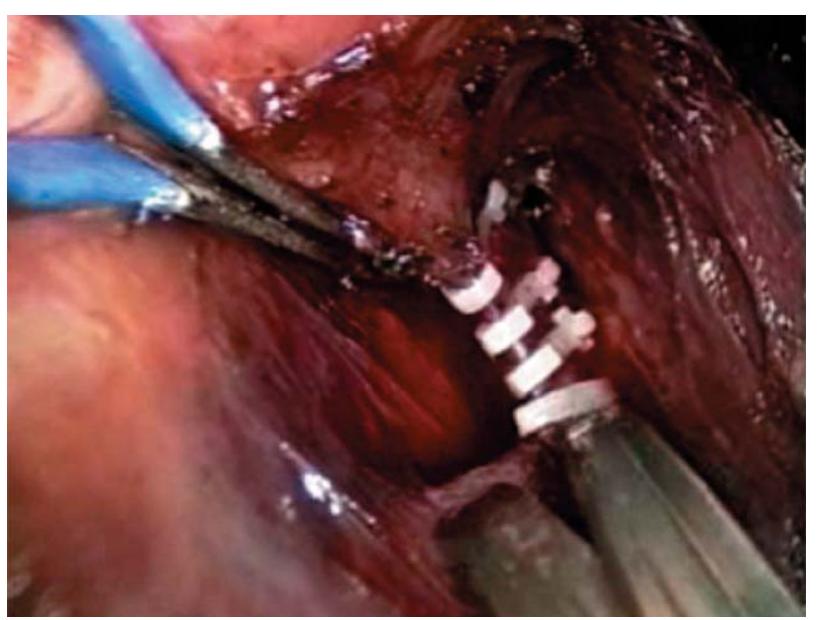

FIGURA 4. Visión endoscópica de la vena adrenal con los clips hemostáticos colocados en un caso de feocromocitoma derecho. 


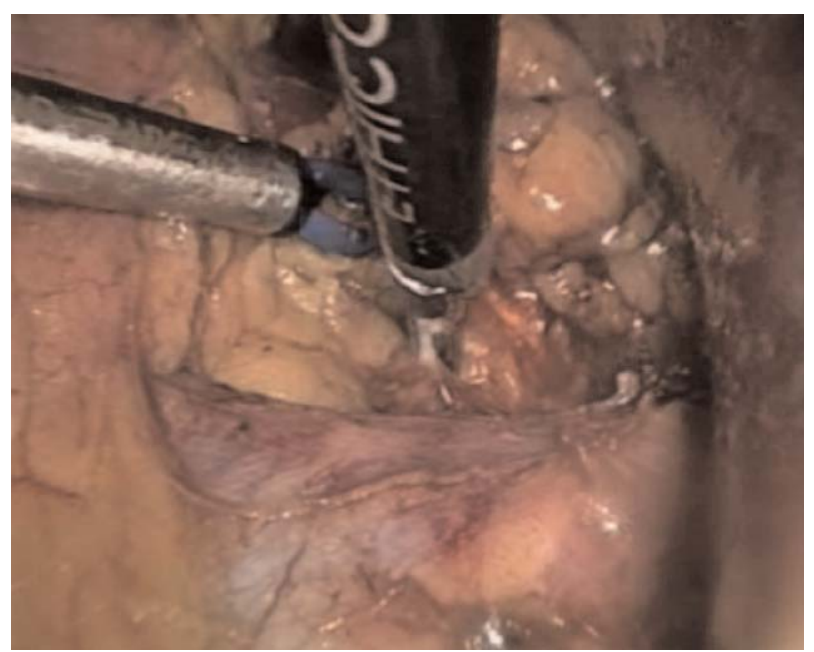

FIGURA 5. Fotografia que muestra el excelente campo de visión de la pared lateral de la vena cava que nos permite la óptica de $30^{\circ}$.

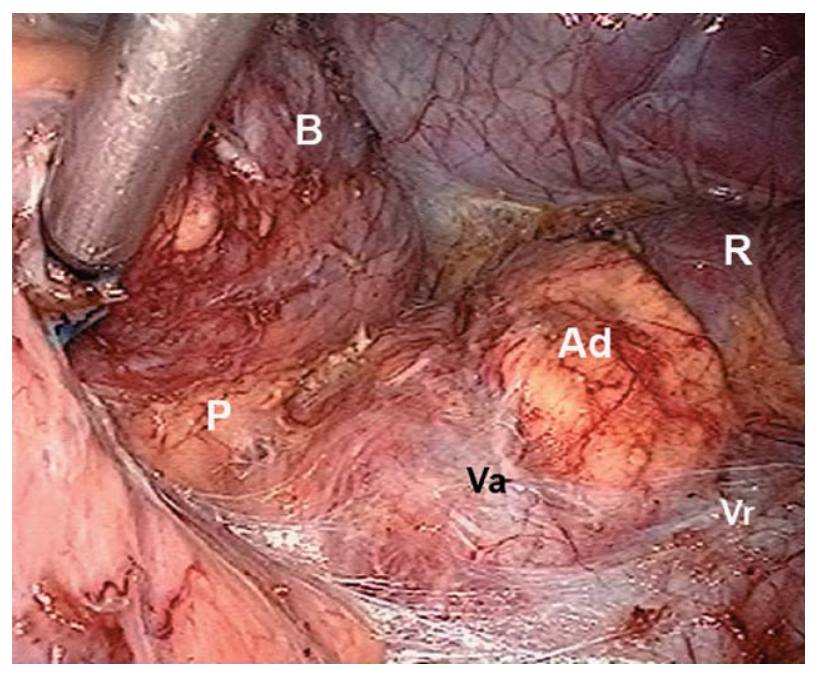

FIGURA 6. Visión laparoscópica del territorio de la adrenal izquierda, una vez realizada la maniobra de luxación del bazo. (B) bazo, (R) riñón, (Ad) adrenal, (P) cola del páncreas, (Va) vena adrenal, (Vr) vena renal.

Análisis estadístico: Los datos vienen expresados como Media \pm DE. La comparación de medias se ha realizado con el U-test de Mann-Whitney y/o la t-student según los casos, considerando significativa una $\mathrm{p}<0,05$.

\section{RESULTADOS}

La cirugía se pudo completar en todos los casos menos en una paciente con enfermedad de Cushing e I.M.C. (Índice de Masa Corporal) de 40, al comienzo de la serie. Las características demográficas, operatorias y patológicas de la serie vie-

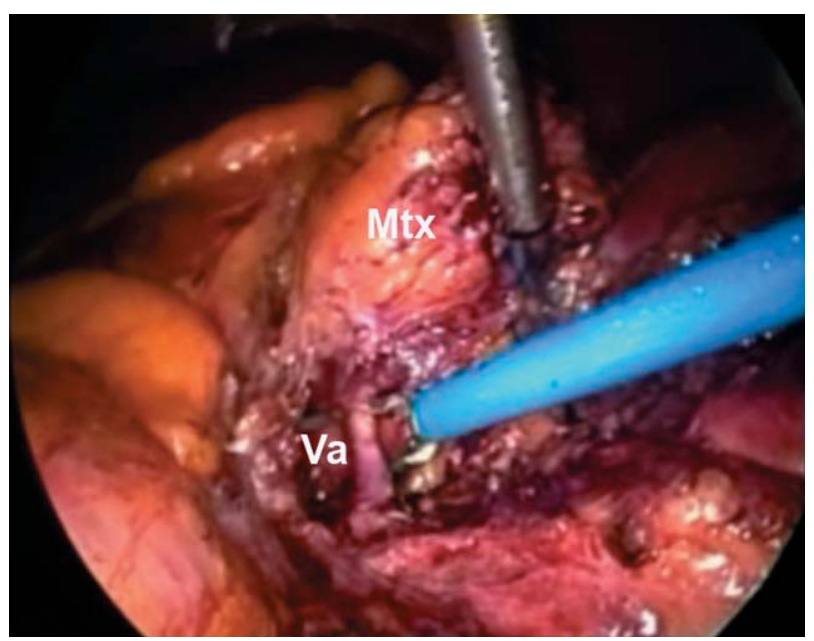

FIGURA 7. Disección de la vena adrenal (Va) en un caso de metástasis adrenal por adenocarcimoma pulmonar (Mtx).

nen detalladas en la Tabla 1. El tiempo quirúrgico medio para toda la serie fue de $182,5(97,75)$ minutos (rango: 59-400), con diferencias cuando se comparan los primeros casos de la serie, donde el tiempo quirúrgico fue más prolongado debido fundamentalmente a la curva de aprendizaje, inevitable en este tipo de cirugía. Hemos dividido los resultados en 2 períodos:

a) Inicial (1998-2002, $\mathrm{n}=12$ ) con un tiempo quirúrgico medio de 261,6 $(76,61)$ minutos (rango: 150-400) y,

b) de consolidación de la técnica (2003-2006, $\mathrm{n}=12$ ) con un tiempo quirúrgico medio de 103,3 $(20,92)$ minutos (rango: 59-150), con una diferencia altamente significativa $(\mathrm{p}<0,001)$ entre ambos grupos (Fig. 8).

El sangrado medio en 23 procedimientos fue de 43,26 (25) cc (rango: 10-100). En el caso 9 se produjo una pérdida hemática de $600 \mathrm{cc}$ sin precisar transfusión postoperatoria. Se produjeron 2 complicaciones intraoperatorias menores: una perforación hepática producida por una de las $<$ palas del retractor laparoscópico, al principio de la serie, y una laceración de la cápsula esplénica, resueltas ambas de forma intracorpórea, mediante coagulación y colocación de material hemostático. Aunque actualmente no dejamos drenaje de forma rutinaria, en los casos en que se colocó $(n=16)$, éste fue retirado entre 12 y $24 \mathrm{~h}$. del postoperatorio. La sonda nasogástrica y vesical fue retirada en todos los casos al término de la cirugía. El inicio de dieta oral fue de promedio 1,87 
Tabla 1

Datos demográficos y resultados de las adrenalectomías laparoscópicas

\begin{tabular}{|c|c|c|c|c|c|c|c|c|c|}
\hline $\begin{array}{c}\text { No } \\
\text { Paciente }\end{array}$ & Sexo & Edad & $\begin{array}{l}\text { Diagnóstico clínico } \\
\text { y patológico. }\end{array}$ & Lado & $\begin{array}{l}\text { Diám } \\
\text { (cm) }\end{array}$ & $\begin{array}{l}\text { Peso } \\
\text { (g) }\end{array}$ & $\begin{array}{l}\text { T.Quir } \\
\text { (min) }\end{array}$ & $\begin{array}{l}\text { Sangrado } \\
\text { (cc) }\end{array}$ & $\begin{array}{l}\text { E.M. } \\
\text { (dias) }\end{array}$ \\
\hline 1 & M & 37 & Cushing/adenoma & $\mathrm{D}$ & 2,5 & 11 & 360 & 100 & 5 \\
\hline $2 \#$ & M & 45 & Cushing/adenoma & $\mathrm{D}$ & 1,3 & 8 & 240 & 100 & 5 \\
\hline 3 & $\mathrm{H}$ & 50 & Cushing/hiperplasia & I & 4 & 40 & 300 & 80 & 5 \\
\hline 4 & M & 43 & Incidental/adenoma & I & 2,8 & 20 & 400 & 90 & 5 \\
\hline 5 & M & 38 & Cushing/adenoma & $\mathrm{D}$ & 3,2 & 26 & 170 & 50 & 3 \\
\hline 6 & M & 78 & Feocromocitoma & $\mathrm{D}$ & 3,5 & 30 & 320 & 70 & 5 \\
\hline 7 & M & 53 & Feocromocitoma & I & 5 & 39 & 300 & 100 & 4 \\
\hline 8 & M & 59 & Conn/adenoma & I & 2,7 & 45 & 240 & 40 & 4 \\
\hline 9 & $\mathrm{H}$ & 34 & Feo/Pseudoquiste & $\mathrm{D}$ & 3,5 & 16 & 240 & 600 & 4 \\
\hline 10 & M & 40 & Conn/adenoma & $\mathrm{D}$ & 2,6 & 15 & 180 & 70 & 4 \\
\hline 11 & $\mathrm{H}$ & 66 & Incidental/adenoma & $\mathrm{D}$ & 6 & 67 & 240 & 50 & 4 \\
\hline 12 & M & 41 & Feocromocitoma & $\mathrm{D}$ & 4 & 37 & 150 & 20 & 3 \\
\hline 13 & M & 37 & Conn/adenoma & I & 2 & 19 & 115 & 20 & 3 \\
\hline 14 & M & 27 & Feocromocitoma & I & 1,3 & 20 & 110 & 20 & 3 \\
\hline $15^{*}$ & $\mathrm{H}$ & 67 & Cushing/hiperplasia & $\mathrm{D}$ & 2 & 11 & 120 & 20 & 3 \\
\hline $16^{*}$ & $\mathrm{H}$ & 67 & Cushing/hiperplasia & I & 3 & 13 & 120 & 10 & 3 \\
\hline 17 & $\mathrm{H}$ & 59 & Mtx. ca. pulmón & I & 3 & 52 & 90 & 15 & 3 \\
\hline 18 & M & 60 & Feocromocitoma & $\mathrm{D}$ & 5,3 & 92 & 95 & 10 & 3 \\
\hline 19 & $\mathrm{H}$ & 49 & Feocromocitoma & $\mathrm{D}$ & 4,5 & 48 & 150 & 30 & 3 \\
\hline 20 & M & 60 & Feocromocitoma & $\mathrm{D}$ & 2,5 & 20 & 90 & 20 & 3 \\
\hline $21 \dagger$ & M & 24 & Feocromocitoma & $\mathrm{D}$ & 2 & 11 & 100 & 25 & 3 \\
\hline $22 \dagger$ & M & 24 & Feocromocitoma & I & 1,3 & 11 & 70 & 15 & 3 \\
\hline 23 & $\mathrm{H}$ & 70 & Mtx. ca. pulmón & I & 3,5 & 52 & 90 & 20 & 3 \\
\hline 24 & M & 51 & Conn/adenoma & I & 2,5 & 20 & 59 & 20 & 3 \\
\hline
\end{tabular}

$*, \dagger 2$ pacientes con adrenalectomía bilateral.

\# 1 reconversión.

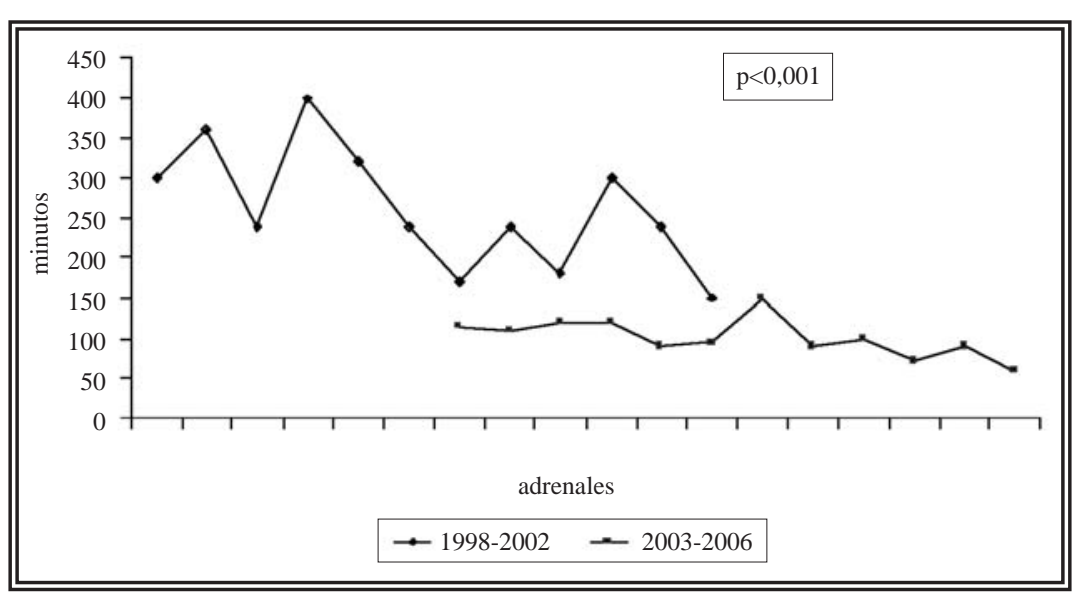

FIGURA 8. Representación lineal de los tiempos quirúrgicos empleados en cada procedimiento, agrupados en 2 etapas cronológicas definidas, con el mismo número de casos ( $n=12 \times 2$ ), observando una diferencia altamente significativa entre ambas.
$(0,94)$ días (rango: 1-4). La medicación analgésica postoperatoria necesaria fue de 32 (21) mg de morfina equivalente (rango: 10-67). La estancia media hospitalaria para todo el grupo fue de 3,62 $(0,82)$ días (rango: 3-5). En los primeros 12 casos, la estancia media fue de 4,25 $(0,73)$ días (rango: $3-5)$ y en los 12 últimos fue de 3 días, con una diferencia significativa $(\mathrm{p}<0.001)$ entre ambos grupos. El diagnóstico anatomopatológico de las piezas resecadas fueron: 9 adenomas corticales, 9 feocromocitomas, 3 hiperplasias nodulares, 2 metástasis de carcino- 
ma de pulmón y 1 pseudoquiste suprarrenal (diagnosticado inicialmente como feocromocitoma, ver Tabla 1).

En los dos casos operados por metástasis, el tumor primario fue un carcinoma de pulmón. El caso 17 presentaba en el momento de la cirugía una fístula broncógena derecha secundaria a la cirugía de exéresis pulmonar, que no presentó problemas durante la realización de la cirugía laparoscópica, siendo el diagnóstico anatomopatológico de la pieza de metástasis de adenocarcinoma poco diferenciado de pulmón, con una supervivencia de 21 meses tras cirugía adrenal y falleciendo por complicaciones pulmonares en el contexto de enfermedad neoplásica diseminada. En el caso 23 el análisis patológico mostró la presencia de metástasis de carcinoma de célula grande de pulmón con desbordamiento de la glándula e invasión del tejido adiposo circundante, con múltiples invasiones vasculares, venosas y arteriales. Con un seguimiento de tres meses tras cirugía adrenal el paciente se encuentra en tratamiento quimioterápico adyuvante, sin evidencia de recidiva local.

\section{DISCUSIÓN}

A la vista de los resultados obtenidos en las grandes series publicadas ${ }^{(2-4)}$, la adrenalectomía laparoscópica representa un indudable avance en el tratamiento de los tumores suprarrenales hormonalmente activos, habiendo demostrado ser una técnica segura, efectiva y reproducible. El beneficio más significativo lo encuentra el paciente en el período postoperatorio cuando lo comparamos con la cirugía abierta, ya que obtiene todas las ventajas de la cirugía minimamente invasiva, como son: menor dolor postoperatorio y consecuentemente menor necesidad de analgésicos, menor estancia hospitalaria, mejor resultado cosmético y más rápida incorporación a la actividad laboral ${ }^{5}$.

La vía elegida en nuestra serie para el abordaje laparoscópico de las adrenales ha sido la vía transperitoneal en todos los casos por considerarla un acceso cómodo, estando familiarizados con las relaciones anatómicas de las distintas estructuras nobles en ambos lados, lo que facilita las maniobras de disección y exéresis del tumor, aspectos que ya han sido previamente reconocidos por otros autores ${ }^{6,7}$. Otra opción de acceso laparoscópico a las adrenales es la vía retroperitoneal, con ventajas e inconvenientes con respecto al abordaje transperitoneal y generalmente en dependencia de la experiencia del cirujano en ésta vía ${ }^{8}$. En fechas recientes se ha descrito el abordaje transdiafragmático, que se encuentra en fase de desarrollo de la técnica y de sus indicaciones ${ }^{9}$. También se ha comunicado un abordaje supragástrico de la adrenal izquierda en el transcurso de la cirugía bariátrica ${ }^{10}$. Obviamente estos abordajes estarían limitados a casos muy concretos y a cirujanos con una gran experiencia laparoscópica.

La posición y número de trocares utilizados en la adrenalectomía derecha es similar a la descrita por otros autores ${ }^{4,7}$ aunque cuando abordamos la adrenal izquierda sólo precisamos colocar tres puertos de trabajo, ya que de forma sistemática luxamos el bazo medialmente, maniobra ya descrita previamente ${ }^{11}$, que nos permite una amplia exposición del territorio de la adrenal y de las estructuras vecinas, por lo que no es necesario el uso de retractores laparoscópicos en ese lado.

En los pacientes con feocromocitoma, el acceso temprano a la vena adrenal y su ligadura con 2 o 3 clips es prioritario con el fin de evitar las posibles crisis hipertensivas intraoperatorias derivadas de la manipulación tumoral, de forma similar a como se hace en cirugía abierta. En los 9 casos operados por nosotros no se produjeron alteraciones cardiovasculares importantes ni en la realización del pneumoperitoneo ni en las maniobras de disección de la vena adrenal, encontrando esta técnica segura tanto para la exéresis completa como para la resección parcial $^{12-14}$. Un paciente que fue diagnosticado previamente de un feocromocitoma quístico debido a una clinica de cefaleas intensas, palpitaciones y cifras de catecolaminas elevadas que respondió a tratamiento con $\alpha$-bloqueantes, presentó en el análisis patológico de la pieza la presencia de un pseudoquiste adrenal; el estudio inmunohistoquímico complementario reveló un linfangioma como patología de base, sin encontrarse evidencia de feocromocitoma. Los pacientes con enfermedad de Cushing presentaron en general una mayor proporción de la grasa intraabdominal 
como hecho característico del sindrome ${ }^{15}$ observando en este grupo de patología la única reconversión de la serie, motivada por una gran distancia de la piel hasta el retroperitoneo que dejaba corto el material laparoscópico, siendo imposible completar la exéresis con esta técnica. Es en este tipo de pacientes, con cuadros de obesidad mórbida, donde tendría una de sus indicaciones el abordaje retroperitoneal de la adrenal ${ }^{8}$. Los pacientes que presentan hiperaldosteronismo primario son una indicación ideal para la adrenalectomía laparoscópica, ya que se trata en general de tumores pequeños, con escasas posibilidades de malignización y sin riesgos de crisis hipertensivas como en el caso de los feocromocitomas, por lo que su abordaje quirúrgico no suele plantear problemas ${ }^{4,6}$. Aunque se han realizado adrenalectomías parciales en casos de afectación suprarrenal bilateral por diversas patologias generalmente hereditarias ${ }^{6,16}$, en los dos pacientes en los que realizamos adrenalectomía bilateral no se contempló la posibilidad de resección parcial después de valoración con los endocrinos y los propios pacientes, ante la hipotética recidiva tumoral sobre todo en el caso de MEN II.

La patología tumoral maligna de las adrenales se está tratando actualmente con esta técnica, aunque de forma muy selectiva ${ }^{17}$. Las metástasis suprarrenales así como carcinomas adrenales muy seleccionados son opciones que se consideran actualmente tributarias de cirugía laparoscópica siempre que el tumor se encuentre confinado en la glándula en los estudios de imagen realizados (TAC, RMN) y se respeten los principios de la cirugía oncológica, debiendo realizar reconversión en el caso de infiltración local ${ }^{18-20}$. En nuestra experiencia, en dos casos con metástasis suprarrenal de carcinoma de pulmón, la cirugía laparoscópica se realizó sin incidencias, incluso en el paciente cuya anatomía patológica mostraba afectación extraglandular, siendo para ello de gran ayuda el empleo del bisturí armónico.

En una revisión de la literatura sobre 1000 adrenalectomías laparoscópicas, la tasa global de complicaciones (incluyendo procesos benignos y malignos) osciló entre un 10 a 45\%, y el número de reconversiones entre un 5 a 20\% . En la serie presentada por nosotros, la tasa de complicaciones menores y de reconversiones fue algo inferior a las publicadas en la literatura.
La curva de aprendizaje es demandante, al igual que en otros procedimientos de cirugía laparoscópica, algo mayor en la adrenalectomía izquierda debido fundamentalmente a la disección más amplia que hay que hacer con respecto al lado derecho, aunque no es necesario un gran número de casos para adquirir un entrenamiento adecuado. El tiempo operatorio disminuye conforme se incrementa el número de casos tratados, pudiendo afirmar a la vista de los datos expuestos en el presente trabajo que no son necesarios más de 15 casos para obtener un rendimiento quirúrgico adecuado, algo inferior que los propuestos por otros grupos ${ }^{21,22}$.

El papel que la robótica tiene en la cirugía adrenal es un campo emergente, habiéndose publicado los primeros casos realizados completamente con técnica asistida por robot ${ }^{23,24}$, aunque con ciertas limitaciones y problemas derivados de la tecnología actualmente disponible.

$\mathrm{El}$ beneficio obtenido en términos de estancia media, dolor postoperatorio, incorporación temprana a la vida laboral de la cirugía laparoscópica ha sido ampliamente refrendado en numerosos trabajos, proponiendo incluso programas de cirugía laparoscópica ambulatoria con importantes reducciones de costes para los Sistemas Sanitarios Nacionales ${ }^{25}$. Esto obligará en un futuro próximo a rediseñar los programas de aprendizaje quirúrgico de los residentes en técnicas laparoscópicas, siendo preceptivo para ello una estricta organización de los contenidos docentes y validación de los mecanismos de seguridad necesarios para realizar este tipo de técnicas.

\section{CONCLUSIONES}

Presentamos la experiencia adquirida por nuestro grupo en el manejo laparoscópico de la adrenal, patología por desgracia ausente de muchos servicios de urología del país por ser derivada, con gran frecuencia, a los servicios de cirugía general. La adrenalectomía laparoscópica ha demostrado ser el patrón de oro en el tratamiento de la patología tumoral benigna y se está afianzando en el caso de tumores malignos bien seleccionados y cumpliendo estrictamente los criterios que debe presidir cualquier cirugía oncológica. Las ventajas que esta técnica reportan al 
Tabla 2

Adrenalectomía laparoscópica. Consideraciones a propósito de 24 procedimientos (Pascual Piédrola JI et al.)

\begin{tabular}{|l|c|c|}
\hline Cosas para recordar & Adrenal derecha & 3 \\
\hline Trocares aconsejados & 4 & $30^{\circ}$ \\
\hline Óptica adecuada & $30^{\circ}$ & Bdrenal izquierda \\
\hline Material imprescindible & \multicolumn{2}{c|}{\begin{tabular}{c} 
Pisturí armónico bipolares \\
\hline Maniobra útil
\end{tabular}} \\
\hline Consejo & Colocación pinza paraseparar el hígado & Maniobra de luxación esplénica \\
\hline \multicolumn{2}{|c|}{ Ser exquisito en la hemostasia } \\
\hline
\end{tabular}

paciente son probablemente superiores a las obtenidas tras la realización de otros procedimientos laparoscópicos cuando se comparan con su equivalente en cirugía abierta, debido fundamentalmente a la profunda localización anatómica de las adrenales. Los datos presentados en este trabajo nos confirman la bondad del procedimiento, la corta curva de aprendizaje en cuanto al número de casos necesarios y el coste-efectividad de la técnica, tanto en los tiempos de ocupación de quirófano como en la estancia media de los pacientes. Queremos finalmente mostrar, a modo de consejo o recordatorio en la Tabla 2 , los pequeños trucos prácticos que hemos ido aprendiendo a lo largo de nuestra experiencia en este tipo de abordaje.

\section{REFERENCIAS}

1. Gagner M, Lacroix A, Bolte E. Laparoscopic adrenalectomy in Cushing's syndrome and pheochromocytoma. New Engl J Med. 1992;327(14): 1033.

2. Ramacciato G, Paolo M, Pietromaria A, Paolo B, Francesco D, Sergio P, et al. Ten years of laparoscopic adrenalectomy: lesson learned from 104 procedures. Am Surg. 2005;71(4):321-325.

3. Izaki H, Fukumori T, Takahashi M, Taue R, Kishimoto T, Tanimoto S, et al. Indications for laparoscopic adrenalectomy for non-functional adrenal tumor with hypertension: usefulness of adrenocortical scintigraphy. Int J Urol. 2006;13(6):677-681.

4. Zacharias M, Haese A, Jurczok A, Stolzenburg JU, Fornara P. Transperitoneal laparoscopic adrenalectomy: outline of the preoperative management, surgical approach, and outcome. Eur Urol. 2006;49(3): 448-459.

5. Winfield HN, Hamilton BD, Bravo EL, Novick AC. Laparoscopic adrenalectomy: The preferred choice? A comparison to open adrenalectomy. J Urol. 1998;160(2):325-329.

6. Janetschek G, Altarac S, Finkenstedt G, Gasser R, Bartsch G. Technique and results of laparoscopic adrenalectomy. Eur Urol. 1996; 30(4): 475-479.

7. Smith CD, Weber CJ, Amerson JR. Laparoscopic adrenalectomy: New gold standard. Word J Surg. 1999;23(4):389-396.

8. Gasman D, Droupy S, Koutani A, Salomon L, Antiphon P, Chassagnon $\mathrm{J}$, et al. Laparoscopic adrenalectomy: The retroperitoneal approach. J Urol. 1998;159(6):1816-1820.

9. Gill IS, Meraney AM, Thomas JC, Sung GT, Novick AC, Lieberman I. Thoracoscopic transdiaphragmatic adrenalectomy: The initial experience. J Urol. 2001;165(6 Pt 1):1875-1881.
10. Bardaro SJ, Gagner M. Laparoscopic left adrenalectomy during Rouxen-Y gastric bypass using a supragastric approach. Obes Surg. 2006; 16(7):919-923.

11. Pascual JI, Grasa V, Cuesta JA. Adrenalectomía laparoscópica derecha e izquierda. In I. Castillón, editor. Curso Audiovisual de Nuevas Tecnologías en Urología. Módulo IV. Adalia farma, S.L. 2005, pp 15-22.

12. Sprung J, O'hara JF, Gill IS, Abdelmalak B, Sarnaik A, Bravo EL. Anesthetic aspects of laparoscopic and open adrenalectomy for pheochromocytoma. Urology. 2000;55(3):339-343. 13. Janetschek G Finkenstedt G, Gasser R, Waibel UG, Peschel R, Bartsch G, et al Laparoscopic surgery for pheochromocytoma: Adrenalectomy, partial resection, excision of paragangliomas. J Urol. 1998;160(2):330-334.

14. Guerrieri M, Baldarelli M, Scarpelli M, Santini S, Lezoche G, Lezoche E. Laparoscopic adrenalectomy in pheochromocytomas. J Endocrinol Invest. 2005;28(6):523-527.

15. Guazzoni G, Montorsi F, Bocciardi A, da Pozzo L, Rigatti P, Lanzi R, et al. Transperitoneal laparoscopic versus open adrenalectomy for benign hyperfunctioning adrenal tumours: A comparative study. J Urol. 1995; 153(5):1597-1600

16. Kaouk JH, Matin S, Bravo EL, Gill IS. Laparoscopic bilateral partial adrenalectomy for pheochromocytoma. Urology. 2002;60:1100-1103.

17. Lombardi CP, Raffaelli M, De Crea C, Bellantone R. Role of laparoscopy in the management of adrenal malignancies. J Surg Oncol. 2006;94(2): 128-131

18. Miccoli P, Materazzi G, Mussi A, Lucchi M, Massi M, Berti P. A reappraisal of the indications for laparoscopic treatment adrenal metastases. J Laparoendosc Adv Surg Tech A. 2004;14(3):139-145.

19. Porpiglia F, Fiori C, Tarabuzzi R, Giraudo G, Garrone C, Morino M, et al. Is laparoscopic adrenalectomy feasible for adrenocortical carcinoma or metastasis? BJU International. 2004;94(7):1026-1029.

20. Moinzadeh A, Gill IS. Laparoscopic radical adrenalectomy for malignancy in 31 patients. J Urol. 2005;173(2):519-525.

21. Goitein D, Mintz Y, Gross D, Reissman P. Laparoscopic adrenalectomy: ascending the learning curve. Surg Endosc. 2004;18(5):771-773.

22. Eto M, Harano M, Koga H, Tanaka M, Naito S. Clinical outcomes and learning curve of a laparoscopic adrenalectomy in 103 consecutive cases at a single institute. Int J Urol. 2006;13(6):671-676.

23. Moinzadeh A, Gill IS. Robotic adrenalectomy. Urol Clin North Am. 2004;31(4):753-56.

24. Desai MM, Gill IS, Kaouk JH, Matin SF, Sung GT, Bravo EL. Roboticassisted laparoscopic adrenalectomy. Urology. 2002;60(6):1104-1107.

25. Skattum J, Edwin B, Trondsen E, Mjaland O, Raede J, Buanes T. Outpatient laparoscopic surgery: feasibility and consequences on education and health care costs. Surg Endosc. 2004;18(5):796-801.

Correspondencia autor: Dr. J.I. Pascual Piédrola

Servicio de Urología. Hospital de Navarra. Irunlarrea 3.

31008 Pamplona. Tel.: 848422225

e-mail: pascual@pulso.com

Información artículo: Laparoscopia

Trabajo recibido: septiembre 2006

Trabajo aceptado: enero 2007 